\title{
Impact of Facility Radiation Patient Volume on Overall Survival in Patients with Muscle Invasive Bladder Cancer Undergoing Trimodality Bladder Preservation Therapy
}

\author{
Benjamin W. Fischer-Valuck ${ }^{\mathrm{a}, \mathrm{b}, *}$, Soumon Rudra ${ }^{\mathrm{b}}$, Prashant Gabani ${ }^{\mathrm{b}}$, Randall Brenneman ${ }^{\mathrm{b}}$, \\ Ryan Mueller ${ }^{\mathrm{b}}$, Walter Chin ${ }^{\mathrm{a}}$, Hiram A. Gay ${ }^{\mathrm{b}}$, Jeff M. Michalski ${ }^{\mathrm{b}}$, Christopher Abraham ${ }^{\mathrm{b}}$ \\ and Brian C. Baumann ${ }^{\mathrm{b}}$ \\ ${ }^{a}$ Department of Radiation Oncology, Emory University, Winship Cancer Institute, Atlanta, GA, USA \\ ${ }^{\mathrm{b}}$ Department of Radiation Oncology, Washington University in St. Louis, St. Louis, MO, USA
}

\begin{abstract}
.
Background: There is an association between higher hospital and urologist case volume and improved survival outcomes for patients with bladder cancer. Less data on facility volume and outcomes exists following trimodality bladder preservation with TURBT followed by definitive chemoradiation (CRT).

Materials and Methods: The National Cancer Database was queried for patients from 2004-2013 with urothelial bladder cancer (cT2-4aNOM0) receiving definitive CRT after TURBT. We compared OS between high- and low- bladder preservation case volume (BPCV) centers using Cox proportional hazard models. BPCV was dichotomized into high versus low volume at the 70th percentile level (i.e. high volume defined as top 30th percentile of cases and low volume defined as bottom 70th percentile of cases). Propensity matching was performed to match high- and low-volume centers.

Results: A total of 666 treatment facilities treating 1,635 patients with bladder preservation were identified with a median follow-up of 26 months (range, 2-136 months). A 70th percentile cutoff identified 497 patients that received treatment at 64 high-volume facilities. Median OS of patients treated at high BPCV centers was 37.0 months (95\% CI, 27.3-46.7) versus 32.3 months (95\% CI, 27.9-33.6) for patients treated at low BPCV centers $(P=0.004)$. High BPCV facilities were independently associated with a decreased hazard of death $(\mathrm{HR}, 0.86,95 \% \mathrm{CI}, 0.75-0.98 ; P=0.031)$. In the propensity score matched cohort, median OS of patients treated at high BPCV was 36.1 months (95\% CI, 26.5-45.8) versus 28.1 months (95\% CI, 23.9-32.3) for patients treated at a low BPCV facility $(P=0.016)$.

Conclusions: In this observational cohort, treatment at a high BPCV facility was associated with improved OS. Causal factors for this finding may include superior bladder specific clinician expertise, technology, and multidisciplinary-care.
\end{abstract}

\section{INTRODUCTION}

Bladder cancer is the second most common genitourinary malignancy in the United States

${ }^{*}$ Correspondence to: Benjamin W. Fischer-Valuck, MD, Winship Cancer Institute, Emory University, Department of Radiation Oncology, 5665 Peachtree Dunwoody Road NE Atlanta, GA 30342, USA. Tel.: +1 678843 7004; Fax: +1 404778 3760; E-mail: benjamin.w.fischer-valuck@emory.edu.
$[1,2]$. Recommended definitive treatment options for patients with localized muscle-invasive bladder cancer (MIBC) include neoadjuvant chemotherapy followed by radical cystectomy or bladder preservation therapy with maximal transurethral resection of bladder tumor (TURBT) followed by chemoradiation (CRT) [3]. Multiple studies have associated surgeon and hospital volume as independent predictors of survival outcomes in surgically treated cancer 
patients [4-9]. Studies comparing hospital or treatment center volume effects on survival in radiation treated cancer patients suggest similar outcomes $[4$, $10,11]$.

Treatment of MIBC with trimodality bladder preservation therapy requires intensive multidisciplinary coordination between medical oncologists, urologists, radiologists, pathologists, and radiation oncologists. Multiple chemotherapy regimens and protocols are available, and the planning and delivery of pelvic radiation therapy is quite complex. Additionally, CRT for bladder cancer is frequently associated with adverse events that are challenging to manage and an experienced care team is needed to ensure optimal patient outcomes. Given the complexity and multidisciplinary effort required for bladder preservation therapy with definitive CRT, we conducted a National Cancer Database (NCDB) analysis to compare the overall survival of MIBC patients treated with trimodality bladder preservation therapy in high versus low volume facilities. It was our hypothesis that treatment centers with more experience with trimodality bladder preservation therapy would have improved overall survival outcomes compared to lower volume centers.

\section{MATERIALS/METHODS}

\section{Data source and study population}

The NCDB Participant User File was queried for de-identified data from 2004 and 2013 for patients 18-90 years of age diagnosed with urothelial bladder carcinoma [International Classification of Disease for Oncology, 3rd edition (ICD-0-3) codes 8120 and 8130]. The NCDB is a joint program of the American College of Surgeons and the American Cancer Society. Data from approximately $70 \%$ of patients diagnosed at Commission on Cancer (CoC) accredited cancer centers is included and includes patient, tumor, and treatment characteristics. Data elements are collected and submitted to the NCDB from commission-accredited oncology registries using standardized coding and data item definitions such as radiotherapy dose/technique, chemotherapy use/timing, and comorbidity. The Participant User File contains de-identified patient and center information and was exempt from Institutional Review Board review.

We limited our analysis to patients with muscle invasive disease and clinically negative lymph nodes (cT2-4a N0) who received radiation therapy to the bladder or pelvis (Radiation Treatment Volume codes 29 and 34) in conjunction with concurrent chemotherapy (including both single and multi-agent therapy given within 21 days of radiation). Patients with metastatic disease and incomplete treatment data were excluded. All patients underwent TURBT for pathologic diagnosis prior to definitive treatment. Total radiation dose received was defined as the sum of the regional dose delivered plus any boost dose. Patients with total radiation dose $<5040$ cGy or $>7500$ cGy, and less than 20 fractions were excluded from analysis as these radiation doses likely represented either palliative treatment or potential coding errors. Flow chart of patient inclusion is shown in Fig. 1.

The trimodality bladder preservation case volume (BPCV) was calculated as the number of bladder preservation cases completed at each facility over the 10-year time period examined. To create the list of facilities eligible for the study, only patients with one $\mathrm{CoC}$ facility submitting a report were included (PUF_MULT_SOURCE=0). Additionally, only patients with the entire course of radiation completed at the reporting $\mathrm{CoC}$ facility were included (PUF_RAD_LOCATION_OF_RX =1). BPCV was first analyzed as a continuous variable. BPCV was then dichotomized into high versus low volume at the 70th percentile level (i.e. high volume defined as top 30th percentile of cases and low volume defined as bottom 70th percentile of cases). This percentile cutoff corresponded to the top $10 \%$ of facilities classified as high-volume (64 facilities) and $90 \%$ of the facilities as low-volume (602 facilities). A sensitivity analysis was performed using various cutoff percentiles of BPCV (50th, 60th, 80th, 85th, and 90th). To confirm that these facilities were the highest volume in terms of bladder radiation therapy, all cases of bladder radiation (regardless of chemotherapy) were also analyzed, as previously performed for prostate cancer volume analysis [4]. All facilities termed high BPCV were in at least the top 15th percentile of bladder radiation given over the time period (Supplemental Figure 1).

Other covariates included in the analysis included clinical variables such as clinical tumor stage (cT), Charlson Deyo comorbidity, treatment facility type, radiation dose, radiation fractionation, number of chemotherapy agents; and socioeconomic variables such as age, sex, race, year of diagnosis, treatment facility location, insurance status, population setting, household income, and education level. 


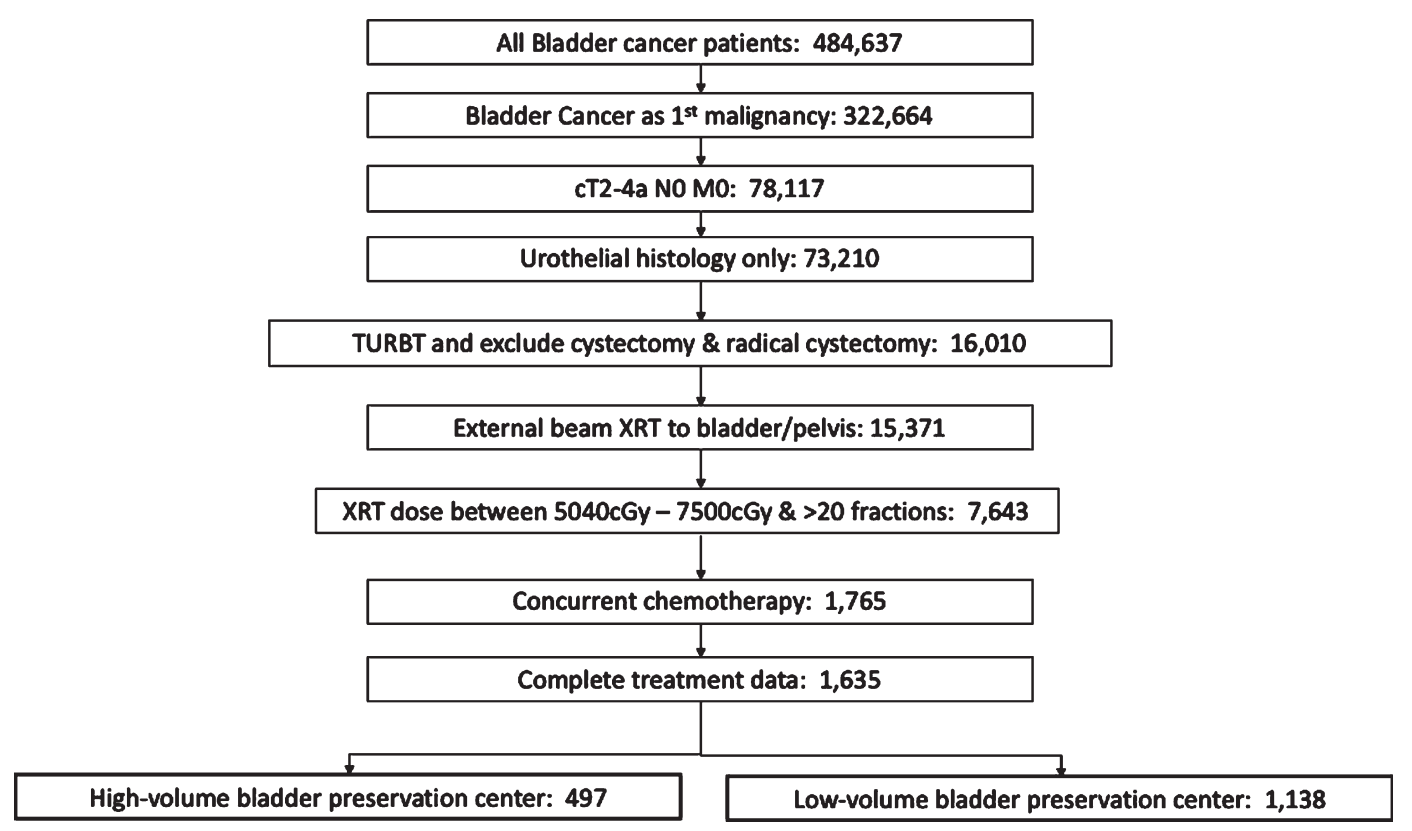

Fig. 1. Consort diagram of inclusion and exclusion criteria. TURBT, transurethral resection of bladder tumor; XRT, radiation therapy.

The primary endpoint of the study was the association between overall survival (OS) and BPCV. To adjust for potential confounding covariables between high and low BPCV centers, OS was also calculated in a propensity score matched cohort. Analysis for patients treated with standard curative fractionated radiation therapy (59.4-64.8 Gy in 33-36 fractions) was also performed.

\section{Statistical analysis}

Overall survival was calculated from diagnosis until death, censoring at last follow-up for patients who were alive. The Kaplan-Meier method was used to estimate overall survival probabilities and the logrank test was used to distinguish survival differences. In addition to these analyses, the clinical and socioeconomic covariables described above were used in univariable analysis. The $\chi^{2}$ test and Fisher's exact test were used to evaluate contingency tables, as appropriate. Variables with $p$-values $<0.05$ on univariate testing were entered into the multivariable analyses using the Cox proportional-hazards model. Propensity score analysis was performed to correct for baseline differences between treatment groups. A (1:1) matching algorithm with 0.2 caliper [12] including the variables used in univariate analysis was used. Significance was considered at a value of $p<0.05$. SPSS Statistics v.23 (IBM Corporation;
Armonk, New York) was used for all statistical analyses.

\section{RESULTS}

\section{Demographics, Patient, Tumor, and Treatment Characteristics}

A total of 666 treatment facilities treating 1,635 patients with bladder preservation were identified with a median follow-up of 26 months (range 2-136 months). A 70th percentile cutoff identified 497 patients that received treatment at 64 high-volume facilities that treated 6 or more cases in the time period examined. 1,138 patients therefore were treated at 602 low-volume facilities (i.e. $<70$ th percentile or $<6$ cases per 10-years). 228 facilities only treated one case in the time period examined. A complete summary of demographic and patient characteristics by treatment volume is provided in Table 1 . An additional dichotomization at the 90th percentile cutoff ( $>=10$ cases per facility in examined time period) identified 172 patients treated at 15 highvolume facilities and 1,463 patients treated at 651 low-volume facilities (Supplemental Table 1).

Patients treated at facilities with higher BPCV were more likely to receive treatment at academic/research centers $(28.6 \%$ versus $23.9 \%, P=0.027)$ and less likely to be located in the central part of the United 
Table 1

Demographics and clinical characteristics

\begin{tabular}{|c|c|c|c|}
\hline & Low volume & High volume & $P$-value \\
\hline Total patient \# (\%) & $1138(69.6)$ & $474(30.4)$ & \\
\hline \multicolumn{4}{|l|}{ Age } \\
\hline Mean (yr) (range) & $76(24-90)$ & $74(32-90)$ & \\
\hline Sex & & & 0.267 \\
\hline Male & $831(73.0)$ & 371 (74.6) & \\
\hline Female & $307(27.0)$ & $433(26.5)$ & \\
\hline Race & & & 0.051 \\
\hline White & $1,028(90.3)$ & $449(90.3)$ & \\
\hline Black & $77(6.8)$ & $29(5.8)$ & \\
\hline Other & $26(2.3)$ & $7(1.4)$ & \\
\hline Unknown & $7(0.6)$ & $12(2.4)$ & \\
\hline Clinical $\mathrm{T}$ stage & & & 0.244 \\
\hline $\mathrm{T} 2$ & $920(80.8)$ & $419(84.3)$ & \\
\hline T3 & $128(11.2)$ & $45(9.1)$ & \\
\hline $\mathrm{T} 4 \mathrm{a}$ & $90(7.9)$ & $33(6.6)$ & \\
\hline Charlson Deyo Comorbidity & & & 0.645 \\
\hline 0 & $751(66.0)$ & $330(66.4)$ & \\
\hline 1 & $270(23.7)$ & $123(24.7)$ & \\
\hline$>1$ & $117(10.3)$ & $44(8.9)$ & \\
\hline Year of Diagnosis & & & 0.999 \\
\hline 2004-2008 & $506(44.5)$ & $221(44.5)$ & \\
\hline 2009-2013 & $632(55.5)$ & $276(55.5)$ & \\
\hline Facility location & & & $<0.0001$ \\
\hline Central & $495(43.5)$ & $143(28.8)$ & \\
\hline Northeast & $259(22.8)$ & $131(26.4)$ & \\
\hline South/Southeast & $207(18.2)$ & $132(26.6)$ & \\
\hline West & $177(15.6)$ & $91(18.3)$ & \\
\hline Setting & & & 0.614 \\
\hline Metropolitan & $871(76.5)$ & $393(79.1)$ & \\
\hline Urban & $190(16.7)$ & $78(15.7)$ & \\
\hline Rural & $14(1.2)$ & $5(1.0)$ & \\
\hline Unknown & $63(5.5)$ & $21(4.2)$ & \\
\hline Facility Type & & & 0.027 \\
\hline Academic/Research Program & $272(23.9)$ & $142(28.6)$ & \\
\hline Non-academic & $866(76.1)$ & $355(71.4)$ & \\
\hline Insurance Status & & & 0.618 \\
\hline Medicaid & $32(2.8)$ & $13(2.7)$ & \\
\hline Medicare & $868(76.3)$ & $363(73.0)$ & \\
\hline Not insured & $21(1.8)$ & $8(1.6)$ & \\
\hline Other government & $18(1.6)$ & $8(1.6)$ & \\
\hline Private & $199(17.5)$ & $105(21.1)$ & \\
\hline Household Income & & & 0.850 \\
\hline$<\$ 30,000$ & $130(11.4)$ & $53(10.7)$ & \\
\hline$\$ 30,000-34-999$ & $217(19.1)$ & $91(18.3)$ & \\
\hline$\$ 35,000-46,000$ & $308(27.1)$ & $144(29.0)$ & \\
\hline$>\$ 46,000$ & $437(38.4)$ & $185(37.2)$ & \\
\hline Unknown & $46(4.0)$ & $24(4.8)$ & \\
\hline Education Level & & & 0.280 \\
\hline$\geq 29 \%$ & $152(13.4)$ & $52(10.5)$ & \\
\hline$\overline{2} 0-28.9 \%$ & $272(23.9)$ & $106(21.3)$ & \\
\hline $14-19.9 \%$ & $287(25.2)$ & $135(27.2)$ & \\
\hline$<14 \%$ & $381(33.5)$ & $180(36.2)$ & \\
\hline Unknown & $46(4.0)$ & $24(4.8)$ & \\
\hline Chemotherapy & & & 0.571 \\
\hline Single agent & $642(56.4)$ & $270(54.3)$ & \\
\hline Double agent & $409(35.9)$ & $192(38.6)$ & \\
\hline Agent \# unknown & $87(7.6)$ & $35(7.0)$ & \\
\hline Radiation Dose & & & 0.181 \\
\hline Median dose (cGy) (range) & $6480(5040-7480)$ & $6480(5040-7380)$ & \\
\hline Median \# fractions & $36(20-44)$ & $36(20-41)$ & \\
\hline
\end{tabular}

High volume $=6$ or more cases per 10-year period ( $>70$ th percentile). Low volume $=5$ or fewer cases per 10 -year period $(<70$ th percentile). 
State $(28.6 \%$ versus $43.3 \%, P<0.0001)$. No differences in age, clinical T-stage, Charlson-Deyo comorbidity, radiation dose, number of chemotherapy agents utilized, or other socioeconomic variables were different between the groups.

\section{Survival outcomes}

The median OS of patients treated at high BPCV centers was 37.0 months (95\% CI, 27.3-46.7) versus 32.3 months (95\% CI, 27.9-33.6) for patients treated at low BPCV centers (Fig. 2a; log rank $P=0.004$ ). After controlling for demographic and clinical factors in Cox proportional hazards modeling, treatment at high BPCV facilities was independently associated with a decreased hazard of death (Hazard ratio (HR), 0.86, 95\% CI, 0.75-0.98; $\log \operatorname{rank} P=0.031$ ) (Table 2). Academic affiliation was not associated with OS (HR: 0.92, 95\% CI, 0.79-1.06) (Table 2). When BPCV was analyzed in this same model as a continuous variable, an increasing number of bladder preservation cases per institute was also independently associated with improved OS (HR: 0.98, 95\% CI, 0.97-0.99; $\log$ rank $P=0.044$ ) (Table 2). In sensitivity analysis, multivariable Cox proportional hazard models in which BPCV was dichotomized at other cutoffs, improved OS was observed at the 75th, 80th, 85 th, and 90th percentiles (Table 3). At the 60th and 50th percentile cut points, there was no OS difference. The median OS of patients treated at very high BPCV centers (90th percentile; $\geq 10$ cases) was 37.7 months (95\% CI, 24.4-34.7) versus 31.9 months (95\% CI, 29.3-34.7) for patients treated at low BPCV centers (Fig. $2 \mathrm{~b} ; P=0.038$ ). In a subgroup analysis, there were 1,213 patients receiving standard curative fractionated radiation therapy $(59.4-64.8 \mathrm{~Gy}$ in $33-36$ fractions) with 897 patients receiving treatment at low BPCV centers and 316 patients at high BPCV centers. The median OS of this subgroup of patients treated at high BPCV centers was 39.1 months $(95 \% \mathrm{CI}$, 29.3-48.8) versus 30.7 months (95\% CI, 27.6-33.7) for patients treated at low BPCV centers (log rank $P=0.009$ ). After controlling for demographic and clinical factors in for this population, treatment at high BPCV facilities remained independently associated with a decreased hazard of death (Hazard ratio (HR), 0.83, 95\% CI, 0.73-0.96; log rank $P=0.037$ )

\section{Propensity matched cohort}

Propensity score matching yielded 487 pairs of patients who were treated at high and low
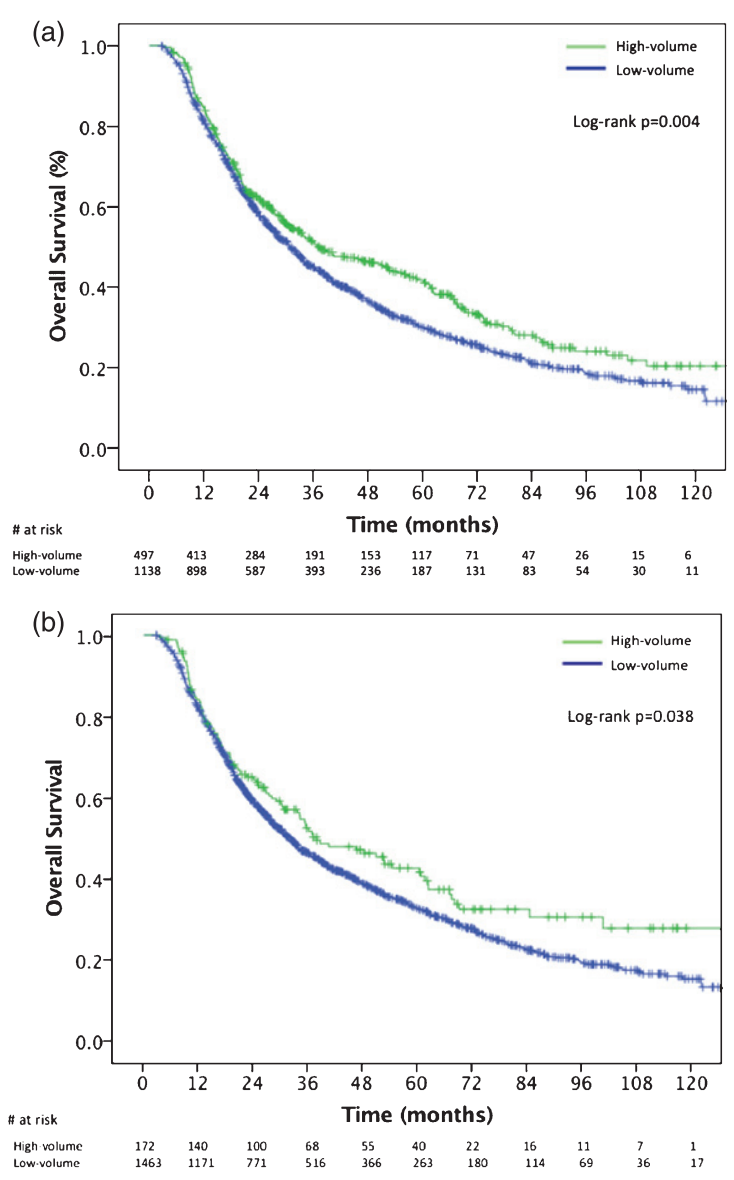

Fig. 2. Kaplan Meier overall survival curves for: (a) patients treated at high-BPCV centers ( $>70$ th percentile; $\geq 6$ cases) versus low-BPCV; (b) patients treated at very high-BPCV centers ( $>90$ th percentile; $\geq 10$ cases) versus low-BPCV. BPCV, bladder preservation case volume.

BPCV centers (974 total patients). After matching, there were no statistically significant differences in any treatment or socioecomonic variables analyzed, including association with academic/research center. The median OS of patients treated at high BPCV was 36.1 months (95\% CI, 26.5-45.8) versus 28.1 months (95\% CI, 23.9-32.3) for patients treated at a low BPCV facility (Fig. 3; log rank $P=0.016$ ). Cox proportional hazards modeling of the propensity score matched cohort showed a similar OS benefit with treatment at high BPCV (HR: 0.82, 95\% CI, $0.70-0.96 ; P=0.016)$.

\section{DISCUSSION}

Hospital and urologist volume have a significant impact on survival outcomes for patients with blad- 
Table 2

Univariate and multivariate analysis

\begin{tabular}{|c|c|c|c|c|}
\hline \multirow{2}{*}{$\begin{array}{l}\text { Analysis } \\
\text { Variable }\end{array}$} & \multicolumn{2}{|c|}{ Univariate Analysis } & \multicolumn{2}{|c|}{ Multivariate Analysis } \\
\hline & HR $(95 \% \mathrm{CI})$ & $P$-value & HR $(95 \% \mathrm{CI})$ & $P$-value \\
\hline \multicolumn{5}{|l|}{ Facility volume } \\
\hline As a continuous variable* & $0.98(0.96-0.99)$ & 0.005 & $0.98(0.97-0.99)$ & 0.044 \\
\hline Low-volume & Reference group & & Reference group & \\
\hline High-volume & $0.82(0.72-0.94)$ & 0.004 & $0.86(0.75-0.98)$ & 0.031 \\
\hline \multicolumn{5}{|l|}{ Age } \\
\hline Continuous & $1.03(1.02-1.03)$ & $<0.0001$ & $1.03(1.02-1.04)$ & $<0.0001$ \\
\hline \multicolumn{5}{|l|}{ Sex } \\
\hline Male & Reference group & & - & \\
\hline Female & $1.05(0.92-1.20)$ & 0.473 & - & \\
\hline \multicolumn{5}{|l|}{ Race } \\
\hline White & Reference group & & - & \\
\hline Nonwhite & $1.09(0.89-1.34)$ & 0.409 & - & \\
\hline \multicolumn{5}{|l|}{ Clinical T stage } \\
\hline T2 & Reference group & & Reference group & \\
\hline T3 & $1.38(1.14-1.65)$ & 0.001 & $1.33(1.11-1.61)$ & 0.003 \\
\hline $\mathrm{T} 4 \mathrm{a}$ & $1.27(1.02-1.59)$ & 0.033 & $1.31(1.05-1.64)$ & 0.019 \\
\hline \multicolumn{5}{|l|}{ Charlson Deyo Comorbidity: } \\
\hline 0 & Reference group & & Reference group & \\
\hline 1 & $1.21(1.05-1.40)$ & 0.008 & $1.22(1.06-1.41)$ & 0.007 \\
\hline$>1$ & $1.77(1.46-2.15)$ & $<0.0001$ & $1.76(1.45-2.14)$ & $<0.0001$ \\
\hline \multicolumn{5}{|l|}{ Year of Diagnosis } \\
\hline 2004-2008 & Reference group & & - & \\
\hline 2009-2013 & $1.05(0.92-1.19)$ & 0.485 & - & \\
\hline \multicolumn{5}{|l|}{ Facility location } \\
\hline Central & Reference group & & - & \\
\hline Northeast & $1.11(0.90-1.31)$ & 0.581 & - & \\
\hline South/Southeast & $1.07(0.86-1.11)$ & 0.675 & - & \\
\hline West & $1.05(0.94-1.09)$ & 0.751 & - & \\
\hline \multicolumn{5}{|l|}{ Setting } \\
\hline Metropolitan & Reference group & - & & \\
\hline Urban & $1.04(0.88-1.22)$ & 0.631 & - & \\
\hline Rural & $1.19(0.69-2.05)$ & 0.535 & - & \\
\hline Unknown & $1.05(0.97-1.21)$ & 0.135 & - & \\
\hline \multicolumn{5}{|l|}{ Facility Type } \\
\hline Academic/Research Program & $0.90(0.77-1.00)$ & 0.050 & $0.93(0.80-1.07)$ & 0.301 \\
\hline Non-academic & Reference group & & Reference group & \\
\hline \multicolumn{5}{|l|}{ Insurance Status } \\
\hline Medicaid & Reference group & & - & \\
\hline Medicare & $1.04(0.92-1.11)$ & 0.682 & - & \\
\hline Not insured & $1.68(0.80-3.33)$ & 0.178 & - & \\
\hline Other government & $1.50(0.82-2.79)$ & 0.222 & - & \\
\hline Private & $0.92(0.75-1.29)$ & 0.685 & - & \\
\hline Unknown & $1.88(0.75-4.25)$ & 0.189 & - & \\
\hline \multicolumn{5}{|l|}{ Household Income } \\
\hline$<\$ 30,000$ & Reference group & - & & \\
\hline$\$ 30,000-34-999$ & $1.07(0.88-1.29)$ & 0.503 & - & \\
\hline$\$ 35,000-46,000$ & $1.05(0.78-1.14)$ & 0.541 & - & \\
\hline$>\$ 46,000$ & $1.05(0.76-1.11)$ & 0.385 & - & \\
\hline Unknown & $1.26(0.84-1.91)$ & .266 & - & \\
\hline \multicolumn{5}{|l|}{ Education Level } \\
\hline$\geq 29 \%$ & Reference group & & - & \\
\hline $20-28.9 \%$ & $1.03(0.84-1.26)$ & 0.804 & - & \\
\hline $14-19.9 \%$ & $0.84(0.70-1.02)$ & 0.086 & - & \\
\hline$<14 \%$ & $0.97(0.79-1.19)$ & 0.738 & - & \\
\hline Unknown & $1.30(0.85-1.99)$ & 0.223 & - & \\
\hline Chemotherapy & & & & \\
\hline Single agent & Reference group & & - & \\
\hline Double agent & $0.91(0.72-1.15$ & 0.443 & - & \\
\hline Agent \# unknown & $1.03(0.82-1.29)$ & 0.810 & - & \\
\hline
\end{tabular}

High volume $=6$ or more cases per 10-year period ( $>70$ th percentile). Low volume $=5$ or fewer cases per 10-year period (<70th percentile). *Cox proportional hazard modeling using facility volume as a continuous variable and excluding the variable low-volume and high-volume in analysis. All other values in the table exclude facility volume as a continuous variable. 
Table 3

Sensitivity analysis

\begin{tabular}{lccc}
\hline $\begin{array}{l}\text { Approximate percentile } \\
\text { for cutoff }\end{array}$ & $\begin{array}{c}\text { Number of Patients }(\%) \\
\text { High-volume }\end{array}$ & $\begin{array}{c}\text { Hazard } \\
\text { Ratio }\end{array}$ & $P$-value \\
\hline 90 th $(\geq 10$ vs $<10$ cases $)$ & $172(10.5 \%)$ & $0.82(0.67-0.98)$ & 0.030 \\
85 th $(\geq 9$ vs $<9$ cases $)$ & $241(14.7 \%)$ & $0.82(0.68-0.97)$ & 0.027 \\
80 th $(\geq 8$ vs $<8$ cases $)$ & $276(16.9 \%)$ & $0.83(0.70-0.98)$ & 0.033 \\
75 th $(\geq 7$ vs $<7$ cases $)$ & $369(22.6 \%)$ & $0.86(0.74-0.99)$ & 0.046 \\
70 th $(\geq 6$ vs $<6$ cases $)$ & $497(30.4 \%)$ & $0.86(0.75-0.98)$ & 0.031 \\
60th $(\geq 5$ vs $<5$ cases $)$ & $635(38.8 \%)$ & $0.90(0.79-1.02)$ & 0.086 \\
50th $(\geq 4$ vs $<4$ cases $)$ & $818(50 \%)$ & $0.92(0.81-1.05)$ & 0.176 \\
\hline
\end{tabular}

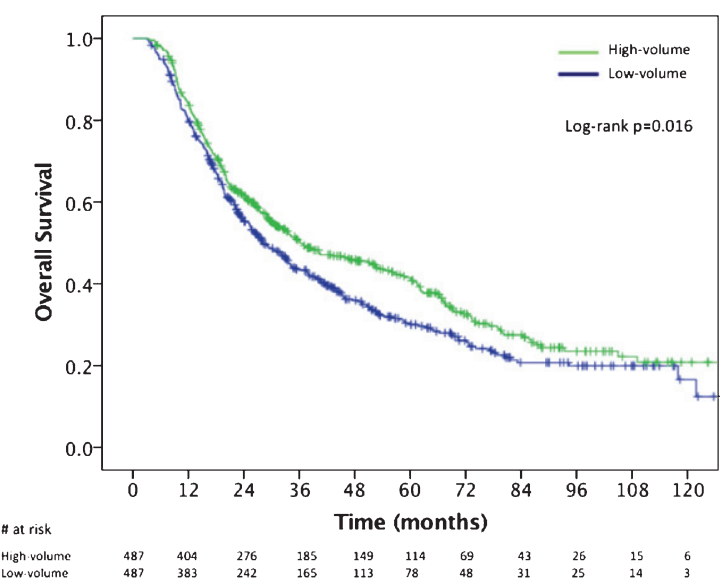

Fig. 3. Kaplan Meier overall survival curves for the propensity score matched cohort comparing patients treated at high-BPCV centers ( $>70$ th percentile; $\geq 6$ cases) versus low-BPCV. BPCV, bladder preservation case volume.

der cancer undergoing radical cystectomy [13, 14]. Higher volume is frequently associated with better survival and shorter hospitalizations for bladder cancer patients [14]. Less is known regarding the relationship between facility volume and survival outcomes for bladder preservation with definitive chemoradiation. In our analysis, we found that a higher number of patients treated with trimodality bladder preservation at a particular facility was associated with increased overall survival. This effect persisted after adjustment for covariables and using propensity matching. Independent of academic affiliation, patients treated with definitive chemoradiation after TURBT for MIBC achieved the longest median overall survival (37.7 months) at centers with experience treating at least one case per year $(\geq 10$ cases in the 10-year period examined). Treatment facilities treating 3 or fewer cases over the time period examined appeared to have worse overall survival compared to centers with more experience. These findings are similar to other studies which demonstrate that patients who receive cancer treatment at lower volume centers have worse survival outcomes $[4,8,15-22]$. The majority of these studies, however, tend to report on the relationship between hospital and surgical volume with outcomes [6, 15-19, 23], and there are fewer studies investigating radiation or concurrent chemoradiation therapy [4, 10, 24].

There are multiple causal explanations to support our results of improved overall survival with more experience treating MIBC with trimodality bladder preservation. Bladder preservation requires a large multidisciplinary team and coordination between multiple oncologic disciplines [25-27]. A high volume facility is more likely to have experts in each of the required disciplines and more frequent multidisciplinary urologic tumor conference to coordinate care. This may translate into patients receiving care in a more consistent approach, including work-up, staging, and treatment, at a high volume center [28-30]. Additionally, high volume facilities have been reported to have higher rates of protocol compliance that translates into improved cancer outcomes $[31,32]$. Bladder preservation therapy is also associated with significant toxicities during and after treatment [33], including radiation-induced cystitis, bowel injury, and hematologic toxicities that require comprehensive support from members of the allied health services, which may be more prevalent at higher volume centers and associated with better outcomes. Also, in terms of chemotherapy, it has been shown that patients treated with systemic therapy at lower volume centers may have a higher mortality rate [34].

There are also specific benefits to high-volume centers with regards to radiation therapy. High-volume facilities are more likely to have radiation oncologists who subspecialize in genitourinary oncology and are more likely to have experience contouring pelvic targets and developing the ideal treatment plan. This subspecialization and experience has been linked to improved survival outcomes in other disease sites $[31,35]$. In a secondary analysis of NRG Oncology 
RTOG 0617 for locally advanced non-small cell lung cancer, treatment at a higher-volume center was associated with less protocol deviations and increased progression-free survival and overall survival [36]. For head and neck radiation therapy, radiation therapy plan quality has been linked to survival outcomes [31]. In the TROG 02.02 trial, centers treating fewer patient were the major source of quality problems and had more noncompliant radiation treatment plans which was related to worse outcomes [31]. In the anal cancer trial RTOG 0529, a large proportion of initial treatment plan submissions required revision, indicating that experienced physicians and treatment centers may benefit patients who are receiving complex radiation treatments [37]. Larger radiation centers, in general, are also more likely to have the most recent treatment technology and adoption of the most recent radiation protocols, which may impact oncologic outcomes. Radiation, especially modern techniques such as IMRT, requires a close collaboration between the physician, dosimetrist, physicist, and therapists to assure that a safe, optimal plan is consistently delivered everyday. Daily imaging and patient set-up are critically important in radiation therapy, particularly with regards to bladder filling, and centers with a higher volume of bladder preservation patients likely have more experience with this daily set-up process. They may also be more likely to use cone-beam CT scans for daily set-up to reduce the risk of marginal miss or excessive dose to bowel. Physicians at higher case volume centers may also be more comfortable dealing with radiation toxicities during therapy, and may have fewer treatment interruptions.

There are limitations in our analysis that may contribute to improved survival seen at high BPCV centers. Survival outcomes may be influenced by selection bias and referral patterns, with patients who have greater access to economic and social resources seeking care at higher volume facilities. In our analysis, however, we found no significant differences in insurance status, income, or education level between high and low BPCV institutes. There is also the possibility that patients initially seen at lower volume centers were referred to institutes with more experience with bladder preservation and such patients are not identifiable in our analysis. Additionally, our results are in contrast to a NCDB study published in 2018 which investigated the impact of academic facility type and case volume on survival for bladder cancer patients undergoing curative intent radiation therapy [38]. These authors found that both academic facility type and case volume were not associated with survival outcomes [38]. In their study, however, nearly half (48\%) of the patients did not receive concurrent chemotherapy. The differences in outcome between their study and ours could be related to the use of systemic therapy, as well as inherent differences in study design and definition of high-volume.

Although the strengths of our study include the large number of patients treated in the modern era of bladder preservation, there are other notable limitations to this retrospective study. Firstly, there are a fairly limited number of bladder preservation cases in the NCDB and even the highest volume center in the analysis only treated 20 cases over the 10 -year period. Additionally, in the current study both cause of death and cancer-specific survival are not available. This is particularly problematic given the multiple comorbidities often facing non-surgical bladder cancer patients and contributes to overall survival. Also, details of the chemotherapeutic agents used and their dosing are not available in the NCDB. Lastly, there is likely a selection bias with respect to treatment center that cannot be fully adjusted for on a multivariable analysis.

\section{CONCLUSION}

In our analysis, we found that treatment at facilities with a higher volume of trimodality bladder preservation patients was associated with improved overall survival. After adjustment for covariables and using propensity matching, this association persisted. As with other disease sites, these findings are likely a result of more tumor site-specific oncologists, greater availability of a multidisciplinary team, more extensive supportive services, higher protocol compliance, and better radiation plan quality.

\section{CONFLICT OF INTEREST}

The authors have no conflict of interest to report.

\section{SUPPLEMENTARY MATERIAL}

The supplementary material is available in the electronic version of this article: http://dx.doi.org/ 10.3233/BLC-190233.

\section{REFERENCES}

[1] Siegel RL, Miller KD, Jemal A. Cancer statistics, 2019. CA Cancer J Clin. 2019;69(1):7-34. 
[2] Fischer-Valuck BW, Rao YJ, Rudra S, Przybysz D, Germino E, Samson P, et al. Treatment Patterns and Overall Survival Outcomes of Octogenarians with Muscle Invasive Cancer of the Bladder: An Analysis of the National Cancer Database. J Urol. 2018;199(2):416-23.

[3] Flaig TW, Spiess PE, Agarwal N, Bangs R, Boorjian SA, Buyyounouski MK, et al. NCCN Guidelines Insights: Bladder Cancer, Version 5.2018. J Natl Compr Canc Netw. 2018;16(9):1041-53.

[4] Chen YW, Mahal BA, Muralidhar V, Nezolosky M, Beard CJ, Den RB, et al. Association Between Treatment at a High-Volume Facility and Improved Survival for RadiationTreated Men With High-Risk Prostate Cancer. Int J Radiat Oncol Biol Phys. 2016;94(4):683-90.

[5] Liu CJ, Chou YJ, Teng CJ, Lin CC, Lee YT, Hu YW, et al. Association of surgeon volume and hospital volume with the outcome of patients receiving definitive surgery for colorectal cancer: A nationwide population-based study. Cancer. 2015;121(16):2782-90

[6] Luchtenborg M, Riaz SP, Coupland VH, Lim E, Jakobsen E, Krasnik M, et al. High procedure volume is strongly associated with improved survival after lung cancer surgery. $\mathbf{J}$ Clin Oncol. 2013;31(25):3141-6.

[7] Schrag D, Cramer LD, Bach PB, Cohen AM, Warren $\mathrm{JL}$, Begg CB. Influence of hospital procedure volume on outcomes following surgery for colon cancer. JAMA. 2000;284(23):3028-35

[8] Bach PB, Cramer LD, Schrag D, Downey RJ, Gelfand SE, Begg CB. The influence of hospital volume on survival after resection for lung cancer. N Engl J Med. 2001;345(3):181-8.

[9] Trinh QD, Bjartell A, Freedland SJ, Hollenbeck BK, Hu JC, Shariat SF, et al. A systematic review of the volumeoutcome relationship for radical prostatectomy. Eur Urol. 2013;64(5):786-98.

[10] Wang EH, Rutter CE, Corso CD, Decker RH, Wilson LD, Kim AW, et al. Patients Selected for Definitive Concurrent Chemoradiation at High-volume Facilities Achieve Improved Survival in Stage III Non-Small-Cell Lung Cancer. J Thorac Oncol. 2015;10(6):937-43.

[11] Verma V, Allen PK, Simone CB, 2nd, Gay HA, Lin SH. Association of Treatment at High-Volume Facilities With Survival in Patients Receiving Chemoradiotherapy for Nasopharyngeal Cancer. JAMA Otolaryngol Head Neck Surg. 2018;144(1):86-9.

[12] Austin PC. Optimal caliper widths for propensity-score matching when estimating differences in means and differences in proportions in observational studies. Pharm Stat. 2011;10(2):150-61.

[13] Konety BR, Dhawan V, Allareddy V, Joslyn SA. Impact of hospital and surgeon volume on in-hospital mortality from radical cystectomy: Data from the health care utilization project. J Urol. 2005;173(5):1695-700.

[14] Goossens-Laan CA, Gooiker GA, van Gijn W, Post PN, Bosch JL, Kil PJ, et al. A systematic review and meta-analysis of the relationship between hospital/surgeon volume and outcome for radical cystectomy: An update for the ongoing debate. Eur Urol. 2011;59(5): 775-83.

[15] Birkmeyer JD, Finlayson SR, Tosteson AN, Sharp SM, Warshaw AL, Fisher ES. Effect of hospital volume on inhospital mortality with pancreaticoduodenectomy. Surgery. 1999;125(3):250-6.

[16] Begg CB, Cramer LD, Hoskins WJ, Brennan MF. Impact of hospital volume on operative mortality for major cancer surgery. JAMA. 1998;280(20):1747-51.
[17] Bilimoria KY, Talamonti MS, Sener SF, Bilimoria MM, Stewart AK, Winchester DP, et al. Effect of hospital volume on margin status after pancreaticoduodenectomy for cancer. J Am Coll Surg. 2008;207(4):510-9.

[18] Bilimoria KY, Talamonti MS, Wayne JD, Tomlinson JS, Stewart AK, Winchester DP, et al. Effect of hospital type and volume on lymph node evaluation for gastric and pancreatic cancer. Arch Surg. 2008;143(7):671-8; discussion 8.

[19] Chen AY, Fedewa S, Pavluck A, Ward EM. Improved survival is associated with treatment at high-volume teaching facilities for patients with advanced stage laryngeal cancer. Cancer. 2010;116(20):4744-52.

[20] Lin JF, Berger JL, Krivak TC, Beriwal S, Chan JK, Sukumvanich $\mathrm{P}$, et al. Impact of facility volume on therapy and survival for locally advanced cervical cancer. Gynecol Oncol. 2014;132(2):416-22.

[21] Kann BH, Park HS, Lester-Coll NH, Yeboa DN, Benitez V, Khan AJ, et al. Postoperative Radiotherapy Patterns of Care and Survival Implications for Medulloblastoma in Young Children. JAMA Oncol. 2016;2(12):1574-81.

[22] Amini A, Jones BL, Ghosh D, Schefter TE, Goodman KA. Impact of facility volume on outcomes in patients with squamous cell carcinoma of the anal canal: Analysis of the National Cancer Data Base. Cancer. 2017;123(2): 228-36.

[23] Ellison LM, Heaney JA, Birkmeyer JD. The effect of hospital volume on mortality and resource use after radical prostatectomy. J Urol. 2000;163(3):867-9.

[24] Lee MS, Tsai SJ, Lee CC, Su YC, Chiou WY, Lin HY, et al. Higher caseload improves cervical cancer survival in patients treated with brachytherapy. Radiat Oncol. 2014;9:234

[25] El-Achkar A, Souhami L, Kassouf W. Bladder Preservation Therapy: Review of Literature and Future Directions of Trimodal Therapy. Curr Urol Rep. 2018;19(12):108.

[26] Aragon-Ching JB, Werntz RP, Zietman AL, Steinberg GD. Multidisciplinary Management of Muscle-Invasive Bladder Cancer: Current Challenges and Future Directions. Am Soc Clin Oncol Educ Book. 2018(38):307-18.

[27] Wuthrick EJ, Zhang Q, Machtay M, Rosenthal DI, NguyenTan PF, Fortin A, et al. Institutional clinical trial accrual volume and survival of patients with head and neck cancer. J Clin Oncol. 2015;33(2):156-64.

[28] Monson JR, Probst CP, Wexner SD, Remzi FH, Fleshman JW, Garcia-Aguilar J, et al. Failure of evidence-based cancer care in the United States: The association between rectal cancer treatment, cancer center volume, and geography. Ann Surg. 2014;260(4):625-31; discussion 31-2.

[29] Cliby WA, Powell MA, Al-Hammadi N, Chen L, Philip Miller J, Roland PY, et al. Ovarian cancer in the United States: Contemporary patterns of care associated with improved survival. Gynecol Oncol. 2015;136(1):11-7.

[30] Ho VK, Damhuis RA, Hartgrink HH. Adherence to national guidelines for gastric cancer in the Netherlands: A retrospective population-based audit. Int $\mathrm{J}$ Cancer. 2013;132(5):1156-61.

[31] Peters LJ, O’Sullivan B, Giralt J, Fitzgerald TJ, Trotti A, Bernier J, et al. Critical impact of radiotherapy protocol compliance and quality in the treatment of advanced head and neck cancer: Results from TROG 02.02. J Clin Oncol. 2010;28(18):2996-3001.

[32] Ohri N, Shen X, Dicker AP, Doyle LA, Harrison AS, Showalter TN. Radiotherapy protocol deviations and clinical outcomes: A meta-analysis of cooperative group clinical trials. J Natl Cancer Inst. 2013;105(6):387-93. 
[33] Feuerstein MA, Goenka A. Quality of Life Outcomes for Bladder Cancer Patients Undergoing Bladder Preservation with Radiotherapy. Curr Urol Rep. 2015;16(11):75.

[34] Giri S, Pathak R, Aryal MR, Karmacharya P, Bhatt VR, Martin MG. Impact of hospital volume on outcomes of patients undergoing chemotherapy for acute myeloid leukemia: A matched cohort study. Blood. 2015;125(21):3359-60.

[35] Boero IJ, Paravati AJ, Xu B, Cohen EE, Mell LK, Le QT, et al. Importance of Radiation Oncologist Experience Among Patients With Head-and-Neck Cancer Treated With Intensity-Modulated Radiation Therapy. J Clin Oncol. 2016;34(7):684-90.

[36] Eaton BR, Pugh SL, Bradley JD, Masters G, Kavadi VS, Narayan $S$, et al. Institutional Enrollment and Survival
Among NSCLC Patients Receiving Chemoradiation: NRG Oncology Radiation Therapy Oncology Group (RTOG) 0617. J Natl Cancer Inst. 2016;108(9).

[37] Kachnic LA, Winter K, Myerson RJ, Goodyear MD, Willins J, Esthappan J, et al. RTOG 0529: A phase 2 evaluation of dose-painted intensity modulated radiation therapy in combination with 5-fluorouracil and mitomycin-C for the reduction of acute morbidity in carcinoma of the anal canal. Int J Radiat Oncol Biol Phys. 2013;86(1):27-33.

[38] Bajaj A, Martin B, Bhasin R, Hentz C, Block AM, Harkenrider MM, et al. The Impact of Academic Facility Type and Case Volume on Survival in Patients Undergoing Curative Radiation Therapy for Muscle-Invasive Bladder Cancer. Int J Radiat Oncol Biol Phys. 2018;100(4):851-7. 